\title{
THE EFFECT OF TWO ADDITIONAL DRY-LAND ACTIVE WARM-UP PROTOCOLS ON THE 50-M FRONT-CRAWL SWIMMING PERFORMANCE
}

original paper

(c) University School of Physical Education in Wroclaw

DOI: https://doi.org/10.5114/hm.2018.76082

\section{ATHANASIOS A. DALAMITROS ${ }^{1}$, ATHANASIOS VAGIOS ${ }^{1}$, ARGYRIS G. TOUBEKIS ${ }^{2}$, GEORGIOS TSALIS $^{1}$, VICENTE J. CLEMENTE-SUAREZ ${ }^{3}$, VASILIKI MANOU ${ }^{1}$}

${ }^{1}$ Laboratory of Evaluation of Human Biological Performance, School of Physical Education and Sport Sciences, Aristotle University of Thessaloniki, Thessaloniki, Greece

${ }^{2}$ School of Physical Education and Sport Sciences, National and Kapodistrian University of Athens, Athens, Greece

${ }^{3}$ Faculty of Physical Activity and Sport Science, European University of Madrid, Madrid, Spain

\section{ABSTRACT}

Purpose. The purpose of the study was to compare the effectiveness of 2 different dry-land active warm-up protocols on 50-m front-crawl swimming performance, biomechanical variables (stroke rate, stroke length, and stroke index), rate of perceived exertion, and exercise heart rate in swimmers of both genders.

Methods. The total of 10 male and 9 female national-level swimmers completed a standardized 1000-m in-water warm-up protocol followed by a 30-min transition phase and a 50-m front-crawl time-trial. During this 30-min period, each swimmer executed, on different occasions, a protocol consisting of either a dynamic stretching routine (stretch) or a power exercise circuit (power) of equal duration $(\sim 5 \mathrm{~min})$ in a randomized sequence. A control condition (control) including a passive recovery strategy after the in-water warm-up protocol was also analyzed.

Results. An improvement in 50-m time-trial performance was demonstrated in male swimmers after executing the power protocol $(p=0.034)$, while in female swimmers a trend towards faster performance times was revealed after the stretch protocol $(p=0.064)$. Stroke index was improved after the stretch routine only in female swimmers $(p=0.010)$. Stroke rate, stroke length, rate of perceived exertion, and exercise heart rate showed no differences among all the 3 conditions in either gender $(p>0.05)$.

Conclusions. Male and female swimmers respond differently to a power or a dynamic stretching protocol. In addition, the variation in responses to different warm-up conditions highlights the importance of individualizing the dry-land warm-up procedure to promote maximum performance during 50-m front-crawl swimming events.

Key words: warm-up strategies, sprint performance, national-level swimmers

\section{Introduction}

The physiological benefits of warming-up prior to a competition are well established [1]. Generally, warmup strategies comprise either active or passive techniques aiming to elevate muscle or core temperature, inducing metabolic, cardiovascular, and neural changes [2]. Specifically in swimming, the pre-competition warm-up practices prescribed by coaches usually combine in-water and dry-land exercises, while dynamic stretching, including swings of the upper and lower limbs, was the most popular pre-competition dry-land exercise strategy advised by international level swim coaches [3].

Yet, the in-water program constitutes the basic component of a warm-up program as it is believed to increase the 'race readiness' of swimmers [4], as well as to allow familiarization with starting blocks and turning walls. In fact, one study that examined the effectiveness of different dry-land programs on 50-m

Correspondence address: Athanasios A. Dalamitros, Faculty of Physical Education and Sport Science, Aristotle University of Thessaloniki, Thermi Thessalonikis, 57001, Thessaloniki, Greece, e-mail: dalammi@phed.auth.gr

Received: November 2, 2017

Accepted for publication: March 13, 2018

Citation: Dalamitros AA, Vagios A, Toubekis AG, Tsalis G, Clemente-Suarez VJ, Manou V. The effect of two additional dry-land active warm-up protocols on the 50-m front-crawl swimming performance. Hum Mov. 2018;19(3):75-81; doi: https:// doi.org/10.5114/hm.2018.76082. 
A.A. Dalamitros et al., Different dry-land warm-up protocols in swimming

sprint swimming performance compared static stretching versus dynamic activities supplementary to the 'typical' in-water warm-up [5]. In this case, and despite the fact that evidence exists regarding the negative effect of static stretching on sprint running performance [6, 7], no differences between the 2 conditions were revealed, assuming that the prior in-water warmup attenuated any positive or negative effects.

The effect of a warm-up strategy on the subsequent swimming performance is also dependent on the transition phase (i.e., the recovery time between the warmup and the actual race). Previous studies have investigated the existence of an 'optimal window' that allows an acute performance enhancement. According to these reports, time periods of 10 and 20 min evoke faster swimming times, compared with those of $45 \mathrm{~min}$, for a 200-m race $[8,9]$. However, during the transition phase, several situations, such as delays in the competition timetable, prolonged marshalling periods, and pool limitations [3], may result in extending this period to up to $45 \mathrm{~min}$ [9].

In this context, a more recent study that examined the impact of an additional passive (heated jacket) and active (dry-land-based exercise circuits) warm-up strategy, within the transition phase, reported an improved 100 -m front-crawl swimming performance when athletes executed the active routine or with the combination of both strategies in junior [10] and elite level [11] swimmers. Nevertheless, during an actual competition, the use of such garments may be costly for an average swimming club or inapplicable during summer events, especially when conducted in outdoor pools.

According to Rushall [4], the beneficial effects of the in-water warm-up are most likely to be short-lived. Regarding the dry-land warm-up procedure, a supplementary exercise program prescribed by swim coaches usually includes stretching exercises [3] that can be easily executed during the transition phase. Meanwhile, strength or activation exercises eliciting the post-activation-potentiation phenomenon (i.e., a shortterm performance enhancement following a high-intensity conditioning activity) are reported to be relevant to a 50-m swimming event [12]. Hence, it would be interesting to compare the potential for enhanced sprint swimming performance after completing 2 different dry-land programs, one based on dynamic stretching exercises and one including power activities, following a typical in-water warm-up.

To the best of our knowledge, no study up to date has analysed the gender effect of different warm-up exercises on swimming performance. Moreover, some previously published data lack the control condition.
Therefore, the purpose of the current study was to investigate the effectiveness of 2 different active dry-land programs of equal duration, executed in the same period of the transition phase, on the 50-m front-crawl performance in National level competitive swimmers of both genders. On the basis of previous observations regarding the responses after additional dry-land-based exercises and since the participants in our study had a similar competitive level, it was hypothesized that the different conditions applied would have the same positive influence on the 50-m time-trial in both male and female swimmers.

\section{Material and methods}

Through a within-subject design, 10 male (mean \pm $S D$ age: $19.3 \pm 2.2$ years, height: $183.2 \pm 7.4 \mathrm{~cm}$, body mass: $83.8 \pm 10.7 \mathrm{~kg}$, and arm span: $188.3 \pm 7.2 \mathrm{~cm}$ ) and 9 female (age: $21.1 \pm 1.9$ years, height: $171.3 \pm$ $4.9 \mathrm{~cm}$, body mass: $65.2 \pm 9.2 \mathrm{~kg}$, and arm span: $174.6 \pm 5.5 \mathrm{~cm})$ national-level swimmers from different swim clubs were recruited in the period after completing their main competition of the season. The male swimmers were 7 sprinters (50- and 100-m specialists) and 3 middle-distance swimmers (200- and 400-m specialists). Among females, 4 swimmers were sprint specialists and 5 were assigned as middle-distance swimmers. The participants had an age range of 17.4-23.7 years, their competitive experience ranged from 9 to 12 years, and they were all placed within the top 8 national ranking in their respective events. Their maximum test performances for the 50-m front-crawl event corresponded to $\sim 560$ FINA 2016 scoring points for a $50-\mathrm{m}$ pool. The subjects were instructed to maintain their normal diet, avoid any strenuous exercises, and abstain from any alcohol consumption in the week of testing.

\section{Measures}

All the procedures were conducted during the same period (June-July) and in the daytime (9:00-12:00 $\mathrm{h})$, under the same water temperature $\left(26-27^{\circ} \mathrm{C}\right)$ in an outdoor 50-m swimming pool. Upon arriving, the swimmers' anthropometric and training characteristics (years of experience, distance specialty, preferred swimming stroke, best swimming time, and usual pre-competition dry-land warm-up strategy) were recorded. Subsequently, the participants were randomly assigned to different warm-up conditions, consisting of either a dynamic stretching routine (stretch) or power exercises (power). In addition, a third warm-up strategy was included (control). 
Within these 3 testing conditions, separated by $48 \mathrm{hrs}$, each athlete performed a 50-m front-crawl allout test. On the basis of a preliminary study of swim coaches' pre-competition in-water practices, a standardized warm-up with the total volume of 1000-m was structured, entailing: 300-m swim (easy pace); $6 \times$ $50 \mathrm{~m}$ swim starting at 1:15 (pull/kick/drill); $8 \times 25 \mathrm{~m}$ starting at 1:00 $(4 \times 25 \mathrm{~m}: 12.5 \mathrm{~m} 90 \%$ of the $50-\mathrm{m}$ race pace followed by $12.5 \mathrm{~m}$ easy and $4 \times 25 \mathrm{~m}$ vice versa); $2 \times 50 \mathrm{~m}$ starting at 2:00 (25 $\mathrm{m}$ at $100 \%$ of the $50-\mathrm{m}$ race pace followed by $25 \mathrm{~m}$ at an easy pace); 100-m easy swim.

After completing the above-mentioned in-water warm-up and during a 30-min transition phase, each athlete executed 1 of the 2 different dry-land exercise protocols, between 15 and $10 \mathrm{~min}$ prior to the $50-\mathrm{m}$ maximum test; during the control condition, the swimmers remained seated throughout the transition phase (Figure 1).

Members of the research personnel were responsible for controlling the race pace during the in-water warm-up and instructing the swimmers on the appropriate execution of the dry-land protocols. All swim tests were performed at a maximum effort and timed individually by 2 experienced researchers with commercial stopwatches (Seiko S141, Japan). The mean value was used for further analysis. A push start and the front-crawl technique were applied during all inwater tests. In addition, the swimmers were advised to avoid underwater gliding after the push start during all 50-m swimming tests. Heart rate (HR) was determined with a strap (Polar S610 Electro, Kempele, Finland) attached to the participant's chest immedi- ately after the 50-m test. Each 50-m time trial was recorded by a digital video camera $(50 \mathrm{~Hz}$, Nikon, L840, China). The camera was placed in the middle $15 \mathrm{~m}$ of the pool, with reference marks set at 20 and $35 \mathrm{~m}$ [13], allowing stroke rate (time to complete 3 stroke cycles; SR: 3 / average duration of the 3 stroke cycles), stroke length (SL: speed / SR), and stroke index (SI: speed $\times$ SL) to be calculated. After the completion of each 50-m test, the swimmers' perception regarding 'the feel of the water' was also reported.

\section{Dry-land protocols}

The power protocol was repeated twice with a 1-min recovery time and completed in a circuit format. This protocol included $3 \times$ medicine ball $(2 \mathrm{~kg})$ throw downs, $3 \times$ medicine ball $(2 \mathrm{~kg})$ side to side crunches, and $3 \times$ $40-\mathrm{cm}$ box jumps. The swimmers rested for $10 \mathrm{~s}$ between exercises, all executed at maximum effort, as previously proposed [10]. The stretch protocol was composed of 7 different exercises, 3 for the upper body (forward arm circles, bend over lateral arms swings, and bend over opposite arms swings), 2 for the body core (twisting toe touch and arm downside bending), and 2 for the lower body (frontal plane leg swings and frontal plane leg swings with a 90-degree knee angle). All exercises were performed bilaterally, with a 10:10 s work-to-rest ratio, and $45 \mathrm{~s}$ of rest between each group of exercises (upper body, body core, and lower body) was allowed. Both dry-land protocols, performed in the head-to-toe order, lasted approximately $4 \mathrm{~min}$ and $50 \mathrm{sec}$ and were designed to activate the same muscle groups. After each dry-land protocol, the

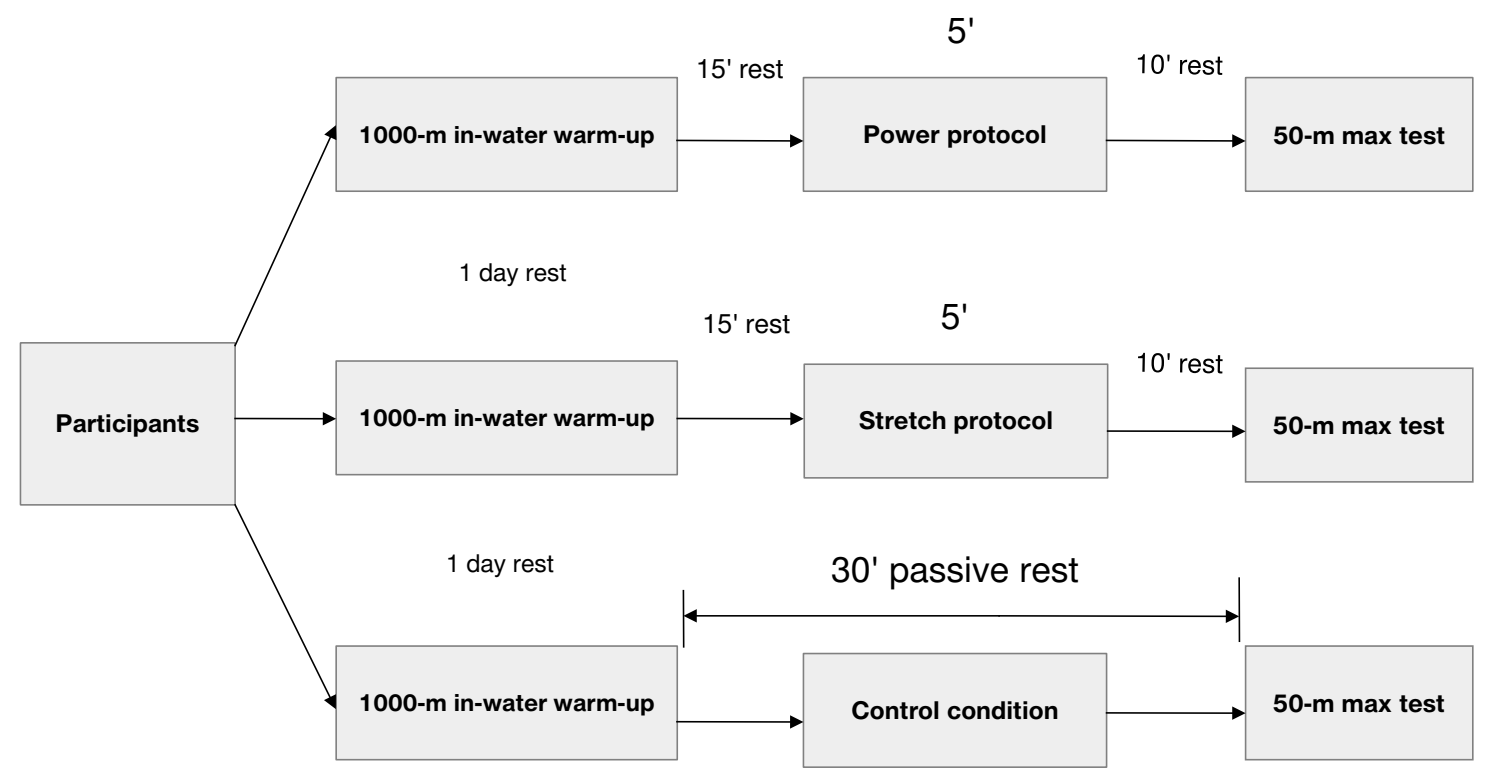

Figure 1. A schematic representation of the experimental design for the 3 testing conditions. 
A.A. Dalamitros et al., Different dry-land warm-up protocols in swimming

rate of perceived exertion (RPE) on a 0-10 scale was recorded.

\section{Statistical analysis}

Data are reported as means $\pm S D$. All statistical tests were processed with the use of the SPSS statistical package (v. 21; SPSS Inc.; Chicago, USA). Normal distribution of the data was tested with the KolmogorovSmirnov test. Sphericity was verified by the Mauchly's test. When the assumption of sphericity was not met, the significance of $F$-ratios was adjusted according to the Greenhouse-Geisser procedure. The analysis of variance for repeated measures with two factors (two-way ANOVA) was applied to compare the 50-m swimming time, SR, SL, SI, RPE, and HR (2 groups [male and female] $\times 3$ warm-up conditions). To explore the effect of the warm-up conditions separately on males and females, we applied a one-way repeated-measures analysis of variance. The Tukey post-hoc test followed when a significant effect was revealed. Cohen's $d$ effect sizes $(d=$ difference between means / pooled $S D)$ were calculated for the difference between the means. The small, medium, and large effects were reflected in values greater than $0.20,0.50$, and 0.80 , respectively [14]. The level of significance was set at $p<0.05$.

\section{Ethical approval}

The research related to human use has been complied with all the relevant national regulations and institutional policies, has followed the tenets of the Declaration of Helsinki, and has been approved by the authors' institutional review board or an equivalent committee.

\section{Informed consent}

Informed consent has been obtained from all individuals included in this study. The form included information about the procedures of the experiment and the right to withdraw at any time. Subjects under the age of 18 provided written parental consent.

\section{Results}

The results of the two-way ANOVA revealed a significant interaction between groups regarding the 50-m test time, where males were faster than females $\left(F_{2,34}=6.66, p=0.004\right)$. The analysis of the biomechanical variables showed that SL during the $50-\mathrm{m}$ test was not different between the groups $\left(F_{2,34}=0.30\right.$, $p=0.74)$. Similar results are reported for SR and SI $\left(F_{2,34}=0.92, p=0.41\right.$ and $F_{2,34}=1.41, p=0.26$, respectively). Neither was HR different between the groups $\left(F_{2,34}=0.48, p=0.62\right)$.

Table 1. Mean $\pm S D$ and $p$ values for the 50-m performance time, SR, SL, SI, HR, and RPE in the 3 warm-up conditions in both genders

\begin{tabular}{|c|c|c|c|c|}
\hline \multirow{2}{*}{ Variable } & \multicolumn{4}{|c|}{ Warm-up condition } \\
\hline & Power & Stretch & Control & $p$ value \\
\hline \multicolumn{5}{|c|}{ Male swimmers $(n=10)$} \\
\hline 50-m time (s) & $26.89 \pm 1.09$ & $27.25 \pm 1.35$ & $27.34 \pm 0.91$ & $0.034 *$ \\
\hline $\mathrm{SR}\left(\right.$ cycles $\left.\cdot \mathrm{s}^{-1}\right)$ & $1.12 \pm 0.06$ & $1.10 \pm 0.08$ & $1.10 \pm 0.07$ & 0.879 \\
\hline $\mathrm{SL}\left(\mathrm{m} \cdot \mathrm{c} \mathrm{ycle}^{-1}\right)$ & $1.74 \pm 0.10$ & $1.68 \pm 0.13$ & $1.67 \pm 0.11$ & 0.879 \\
\hline $\mathrm{SI}\left(\mathrm{m}^{2} \cdot \mathrm{s}^{-1}\right)$ & $3.10 \pm 0.27$ & $3.09 \pm 0.32$ & $3.06 \pm 0.23$ & 0.739 \\
\hline HR (beats $\cdot \min ^{-1}$ ) & $159.9 \pm 11.0$ & $161.0 \pm 9.6$ & $160.0 \pm 12.0$ & 0.390 \\
\hline RPE (0-10) & $2-3$ & $2-3$ & & \\
\hline \multicolumn{5}{|c|}{ Female swimmers $(n=9)$} \\
\hline 50-m time (s) & $31.46 \pm 1.03$ & $30.93 \pm 1.11$ & $31.15 \pm 1.00$ & 0.064 \\
\hline SR $\left(\right.$ cycles $\left.\cdot s^{-1}\right)$ & $0.98 \pm 0.05$ & $0.98 \pm 0.05$ & $0.99 \pm 0.06$ & 0.506 \\
\hline $\mathrm{SL}\left(\mathrm{m} \cdot \mathrm{cycle}^{-1}\right)$ & $1.62 \pm 0.08$ & $1.66 \pm 0.09$ & $1.62 \pm 0.09$ & 0.081 \\
\hline $\mathrm{SI}\left(\mathrm{m}^{2} \cdot \mathrm{s}^{-1}\right)$ & $2.58 \pm 0.16$ & $2.68 \pm 0.19$ & $2.61 \pm 0.17$ & $0.010 *$ \\
\hline HR (beats $\cdot \min ^{-1}$ ) & $163.7 \pm 6.3$ & $165.8 \pm 6.9$ & $162.9 \pm 6.3$ & 0.137 \\
\hline RPE (0-10) & $2-3$ & $2-3$ & & \\
\hline
\end{tabular}

${ }^{*} p<0.05$ between power and stretch

Power - warm-up condition including power exercises; Stretch - warm-up condition including dynamic stretch exercises; Control - warm-up condition including no additional dry-land warm-up; SR - stroke rate; SL - stroke length; SI - stroke index; HR - heart rate after the 50-m test; RPE - rate of perceived exertion after the 2 different dry-land conditions. 


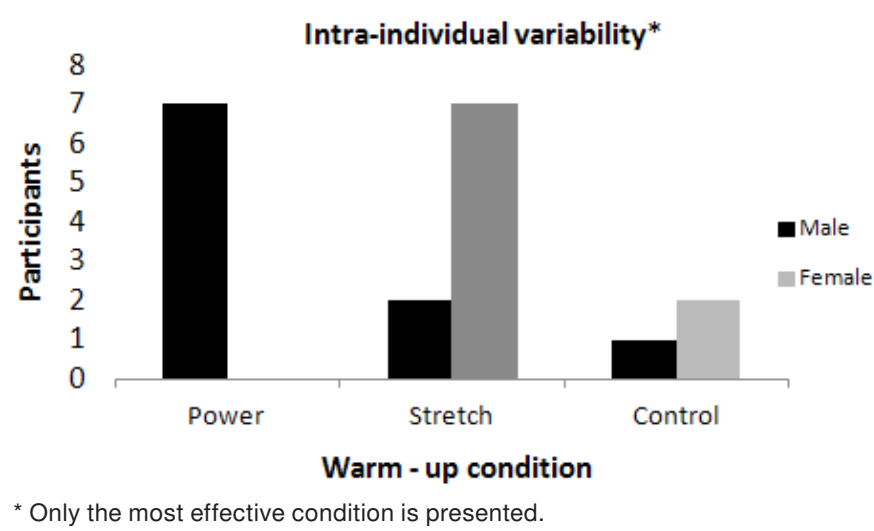

Figure 2. Intra-individual variability in response to the different warm-up conditions in the 50-m swimming performance in both genders.

According to one-way ANOVA analysis, male swimmers' 50 -m performance time was shorter in the power compared with the control condition $\left(F_{2,18}=4.10, d=\right.$ 0.29 [medium effect], $p=0.034$ ). In female swimmers, no difference was observed between the conditions in the 50-m test time $\left(\mathrm{F}_{2,16}=3.27, p=0.064\right)$. However, the 50-m time tented to be shorter after the stretch compared with the power condition $(d=0.31$ [medium effect], $p=0.064$ ). No difference between the conditions was shown for SR in both male and female swimmers $\left(F_{2,18}=0.13, p=0.88\right.$ and $F_{2,16}=0.71, p=0.51$, respectively). Similar results were reported for SL $\left(F_{2,18}=0.13, p=0.88\right.$ and $F_{2,16}=2.95, p=0.08$ for males and females, respectively). Female swimmers showed increased SI in the stretch compared with the power condition $\left(F_{2,16}=6.25, d=0.41\right.$ [medium effect], $p=0.010$ ), whereas for male swimmers, the latter variable showed no significant variations between the conditions $\left(F_{2,18}=0.31, p=0.74\right)$. Finally, HR was not different in any gender $\left(F_{2,18}=0.99, p=\right.$ 0.39 and $F_{2,16}=2.26, p=0.14$ for males and females, respectively) (Table 1 ).

A further analysis of the data, based on visual inspection, revealed an intra-individual variability in the 50-m swimming performance in both genders (Figure 2).

\section{Discussion}

Power and dynamic stretching exercise warm-up practices are well-accepted by elite swimming coaches [3]. In the current study, a power, dynamic stretch, and control dry-land warm-up practices were applied to examine gender-related differences in 50-m front-crawl performance. Improvement in the performance time was observed in male swimmers after the power protocol compared with the control warm-up strategy. In contrast, no significant difference was obtained for the rest of the variables tested (SR, SL, SI, $\mathrm{HR}$, or RPE). In female swimmers, performance time showed medium improvement after performing the stretch compared with the power protocol. Supporting the beneficial effect of the stretch protocol, SI was enhanced during this protocol compared with the power protocol.

Despite the differentiation in the results between male and female swimmers, the magnitude of swimming performance differences noticed for both genders within the 3 warm-up conditions is considered noteworthy. Interestingly, a $1.70 \%$ performance enhancement for male swimmers after the power protocol compared with the control and a similar improvement of $1.66 \%$ for female swimmers following the stretch compared with the power protocol were revealed. Shorter mean 50-m times were observed in males after the control protocol and in females following the power protocol. These results are partially consistent with those obtained by Moran et al. [5], who reported no significant differences in the 50 -m front-crawl performance time among 16 college-level swimmers of both genders after a static stretching protocol or a dynamic activity routine. However, in the previous study, the different dry-land protocols were preceded by an in-water warm-up, the gender-related differences were not examined, and, moreover, a control condition was not included in the experimental design.

The magnitude of performance differences in the 50 -m front-crawl test reported in the current study can be characterized as meaningful. Indeed, previous studies demonstrated a mean performance improvement during an entire training season of approximately $0.6-1 \%$ in Olympic level swimmers [15, 16]; analogous annual performance enhancement was reported in national-level swimmers of both genders aged $17-21$ years $(1.03$ and $0.57 \%$ for males and females, respectively) [17]. Regarding the biomechanical variables analysed, no significant differences among the 3 warm-up conditions were found in male swimmers. Contrary, in female participants, SI was shown to be higher following the stretch protocol. This different response between the two genders, apparently induced by the different dry-land exercise protocols (also affecting the 50-m performance time), remains an unsolved issue, as we can only hypothesize that genderrelated differences in factors such as muscle mass [18] and flexibility [19] would account for this result. Yet, a possible difference in the movement velocity during the execution of the power protocol may have not in- 
A.A. Dalamitros et al., Different dry-land warm-up protocols in swimming

fluenced the 'goal' activity (i.e., the 50-m swim test), since this factor is not decisive for post-activation potentiation response [20].

Previous studies involving similar dry-land exercises before a short-distance race in swimming implemented the same external loads for both genders, without, however, aiming to examine any possible gender-related differences [10, 11]. In our study, RPE values after the 2 dry-land protocols were similar for both genders (Table 1), suggesting that swimmers acknowledged the same level of effort before the 50-m test. Nevertheless, 3 of the female participants reported a 'poor catch and pull' feeling after the power protocol. This observation should be taken under consideration in future research when analysing different training responses in male and female populations after a power stimulus. It is also important to note that all the 3 maximum swim trials were performed with equal intensity for both genders, as indicated by HR values (Table 1).

The participants also demonstrated an intra-individual variability. Specifically, 2 of the male swimmers performed better after the stretch compared with the power protocol, while 4 swimmers presented shorter performance time following the control compared with the stretch protocol. Finally, 1 male participant swam faster after the control compared with the power protocol. Results were somewhat clearer for female participants: 2 swimmers showed an enhanced performance after the control compared with the stretch protocol, while 2 swimmers swam faster following the power compared with the control condition. No female participant swam faster after the power compared with the stretch protocol (Figure 2). These modifications cannot be attributed to the different distance specialty among the participants in the current study as middle-distance swimmers and sprint swimmers demonstrated similar variations in performance times during the different warm-up conditions.

Taking into consideration the aforementioned data, and in line with previous studies [21, 22], we can propose that swim coaches should analyse the individual response during the pre-competition warm-up to attain maximum gains in sprint performance. Finally, the swimmers' usual pre-competition warm-up strategy, prescribed by different coaches, had no influence on the results, since the majority of participants reported that dynamic stretching was their most preferable strategy.

A potential limitation of our study is the fact that the participants were not all front-crawl specialists. However, this specific swimming technique analysed constitutes the most preferable technique applied dur- ing both training sessions and competitions, enabling the fastest swimming times. Therefore, it is reasonable to suggest that the results presented here are limited to sprint distance events employing the front-crawl technique. A second limitation could be addressed regarding the lack of an electronic time recording system. Nevertheless, in our study, the 2 researchers who were recruited for the task were experienced and certified timekeepers, and they assessed the same participants in each of the three 50-m swim trials. Finally, since the study was conducted in an outdoor swimming pool, a care was taken to ensure that all participants were tested approximately at the same time and environmental conditions on each testing day.

\section{Conclusions}

In summary, we observed that different dry-land strategies following a regular in-water warm-up routine had a different impact on the consecutive 50-m sprint front-crawl performance in national-level swimmers. In addition, these modifications are presented as gender-depended. Male swimmers improved their mean 50-m performance time after the execution of the protocol consisting of power-based exercises. In female swimmers, meaningful performance gains were observed after the dynamic stretching protocol. The current study also suggests the need for analysing the individual responses following different dryland warm-up strategies to achieve an acute enhancement in sprint swimming performance.

\section{Acknowledgements}

The authors would like to thank the swimmers and their coaches who contributed their time in this research. No outside funding was received for this work.

\section{Disclosure statement}

No author has any financial interest or received any financial benefit from this research.

\section{Conflict of interest}

The authors state no conflict of interest.

\section{References}

1. Fradkin AJ, Zazryn TR, Smoliga JM. Effects of warming-up on physical performance: a systematic review with meta-analysis. J Strength Cond Res. 2010;24(1):140148; doi: 10.1519/JSC.0b013e3181c643a0.

2. Bishop D. Warm up II: performance changes following active warm up and how to structure the warm up. Sports Med. 2003;33(7):483-498; doi: 10.2165/0000 7256-200333070-00002. 
3. McGowan CJ, Pyne DB, Raglin JS, Thompson KG, Rattray B. Current warm-up practices and contemporary issues faced by elite swimming coaches. J Strength Cond Res. 2016;30(12):3471-3480; doi: 10.1519/JSC.0000 000000001443.

4. Rushall BS. Warming-up in USRPT. Swim Sci Bull. 2014;51:1-18.

5. Moran MP, Whitehead JR, Guggenheimer JD, Brinkert RH. The effects of static stretching warm-up versus dynamic warm-up on sprint swim performance. J Swim Res. 2014;22(1):1-9.

6. Fletcher IM, Anness R. The acute effects of combined static and dynamic stretch protocols on fifty-meter sprint performance in track-and-field athletes. J Strength Cond Res. 2007;21(3):784-787; doi: 10.1519/R-19475.1.

7. Winchester JB, Nelson AG, Landin D, Young MA, Schexnayder IC. Static stretching impairs sprint performance in collegiate track and field athletes. J Strength Cond Res. 2008;22(1):13-19; doi: 10.1519/JSC.0b013e $31815 \mathrm{ef} 202$.

8. West DJ, Dietzig BM, Bracken RM, Cunningham DJ, Crewther BT, Cook CJ, et al. Influence of post-warm-up recovery time on swim performance in international swimmers. J Sci Med Sport. 2013;16(2):172-176; doi: 10.1016/j.jsams.2012.06.002.

9. Zochowski T, Johnson E, Sleivert GG. Effects of varying post-warm-up recovery time on 200-m time-trial swim performance. Int J Sports Physiol Perform. 2007; 2(2):201-211; doi: 10.1123/ijspp.2.2.201.

10. McGowan CJ, Thompson KG, Pyne DB, Raglin JS, Rattray B. Heated jackets and dryland-based activation exercises used as additional warm-ups during transition enhance sprint swimming performance. J Sci Med Sport. 2016;19(4):354-358; doi: 10.1016/j.jsams.2015. 04.012 .

11. McGowan CJ, Pyne DB, Thompson KG, Raglin JS, Osborne M, Rattray B. Elite sprint swimming performance is enhanced by completion of additional warm-up activities. J Sports Sci. 2017;35(15):1493-1499; doi: 10.1080/02640414.2016.1223329.

12. Sarramian VG, Turner AN, Greenhalgh AK. Effect of postactivation potentiation on fifty-meter freestyle in national swimmers. J Strength Cond Res. 2015;29(4):10031009; doi: 10.1519/JSC.0000000000000708.

13. Neiva HP, Marques MC, Fernandes RJ, Vianna JL, Barbosa TM, Marinho DA. Does warm-up have a beneficial effect on 100-m freestyle? Int J Sports Physiol Perform. 2014;9(1):145-150; doi: 10.1123/ijspp.2012-0345.

14. Cohen J. Statistical power analysis for the behavioral sciences. New York: Lawrence Erlbaum Associates; 1988.

15. Costa MJ, Marinho DA, Reis VM, Silva AJ, Marques MC, Bragada JA, et al. Tracking the performance of worldranked swimmers. J Sports Sci Med. 2010;9(3):411-417.

16. Pyne DB, Trewin CB, Hopkins WG. Progression and variability of competitive performance of Olympic swimmers. J Sports Sci. 2004;22(7):613-620; doi: 10.1080/ 02640410310001655822.
17. Dimakopoulou E. Long term development and prediction models of swimming performance in Greek swimmers, master's thesis. Komotini: Democritus University of Thrace; 2009.

18. Miller AE, MacDougall JD, Tarnopolsky MA, Sale DG. Gender differences in strength and muscle fiber characteristics. Eur J Appl Physiol Occup Physiol. 1993;66(3): 254-262; doi: 10.1007/BF00235103.

19. Kibler WB, Chandler TJ, Uhl T, Maddus RE. A musculoskeletal approach to the preparticipation physical examination. Preventing injury and improving performance. Am J Sports Med. 1989;17(4):525-531; doi: 10.1177/036354658901700413.

20. Seitz LB, Trajano GS, Dal Maso F, Haff GG, Blazevich AJ. Postactivation potentiation during voluntary contractions after continued knee extensor task-specific practice. Appl Physiol Nutr Metab. 2015;40(3):230237; doi: 10.1139/apnm-2014-0377.

21. Neiva HP, Marques MC, Bacelar L, Moínhos N, Morouço PG, Marinho DA. The effect of warm up in short distance swimming performance. Ann Res Sport Phys Act. 2012;3:83-94; doi: 10.14195/2182-7087_3_4.

22. Balilionis G, Nepocatych S, Ellis CM, Richardson MT, Neggers YH, Bishop PA. Effects of different types of warm-up on swimming performance, reaction time, and dive distance. J Strength Cond Res. 2012;26(12): 3297-3303; doi: 10.1519/JSC.0b013e318248ad40. 